\title{
LOG-VEROSSIMILHANÇA COMBINADA PARA COMPARAÇÃO DE ESTRUTURAS DE CONTINUIDADE ESPACIAL EM FLORESTA ESTACIONAL DECIDUAL
}

\author{
Julio Cesar Wojciechowski ${ }^{*}$, Julio Eduardo Arce ${ }^{2}$, Saulo Henrique Weber ${ }^{3}$, \\ Paulo Justiniano Ribeiro Junior ${ }^{4}$, Carlos Alberto da Fonseca Pires ${ }^{5}$ \\ ${ }^{1 *}$ Universidade do Estado do Mato Grosso, Departamento de Engenharia Florestal, Alta Floresta, Mato Grosso, Brasil - \\ juliocw@unemat.br; \\ ${ }^{2}$ Universidade Federal do Paraná, Departamento de Ciências Florestais, Curitiba, Paraná, Brasil - jarce@ufpr.br; \\ ${ }^{3}$ Pontifícia Universidade Católica, São Jose dos Pinhais, Paraná, Brasil - sauloweber@ gmail.com; \\ ${ }^{4}$ Universidade Federal do Paraná, Departamento de Estatística, Curitiba, Paraná, Brasil - paulojus@ufpr.br; \\ ${ }^{5}$ Universidade Federal de Santa Maria, Departamento de Geologia, Santa Maria, Rio Grande do Sul, Brasil - calpires@terra.com.br
}

Recebido para publicação: 30/07/2014 - Aceito para publicação: 17/08/2015

\begin{abstract}
Resumo
O presente estudo teve como objetivo verificar a dependência espacial e distribuição do volume em três fragmentos de Floresta Estacional Decidual, geograficamente separados e com idades pósintervenção distintas, utilizando um modelo geoestatístico único ou combinado. Os dados foram coletados em 56 unidades amostrais de $250 \mathrm{~m}^{2}$, distribuídas sistematicamente em uma malha de $40 \times 40 \mathrm{~m}$, onde foram medidos os indivíduos com DAP $\geq 10 \mathrm{~cm}$ a partir do centro da unidade conforme metodologia descrita por Prodan. Os dados foram submetidos a dois tipos de análise, sendo o primeiro um ajuste individual das áreas a título de comparação entre seus modelos e o segundo, um ajuste proposto pelo método combinado, ambos utilizando modelos geoestatísticos, com ajuste pela função da maximização do logaritmo da verossimilhança. Os modelos foram comparados pelo critério de informação de Akaike (AIC) e a relação do parâmetro alcance como indicação do grau de dependência espacial. Os resultados mostram que os modelos combinados foram superiores em relação aos ajustes dos modelos para as áreas individuais. Indica-se a aplicação de modelos geoestatísticos de log-verossimilhança combinados em formações florestais fragmentadas para uma melhor análise e detecção da estrutura de correlação espacial do volume.
\end{abstract}

Palavras-chave: Geoestatística; inventário florestal; modelos mistos; critério de Akaike.

\begin{abstract}
Combined log-likelihood for comparison of spatial continuity structures in deciduous forest. The present study aimed to examine spatial dependence and distribution of volume in three fragments of Subtropical forest, geographically separated and the different post-intervention ages, using a single geostatistical model or combined model. Data were collected from 56 sampling units of $250 \mathrm{~m}^{2}$ systematically distributed in a grid of $40 \times 40 \mathrm{~m}$. Trees with $\mathrm{DBH} \geq 10 \mathrm{~cm}$ were measured according to Prodan's methodology. Two types of analysis were applied to the data. The first one was an individual adjustment for comparison between their models and the second one consisted in the proposed combined approach adjustment. Both analysis used geostatistical models with adjustment function maximizing log-likelihood. Models were compared using Akaike criterion (AIC) and relational range parameter as an indication of spatial dependence degree. Results show that combined models had lower AIC values as well as greater spatial dependence degree on adjustments of individual areas models. This research indicates the use of combined log-likelihood geostatistical models to study fragmented forests for analysis and detection of spatial volume correlation structure. Keywords: Geostatistics; forest inventory; mixed models; Akaike criterion.
\end{abstract}

\section{INTRODUÇÃO}

Modelos geoestatísticos baseados em ajustes por máxima verossimilhança apresentam vantagens em relação aos métodos tradicionais de inferência espacial, dentre as principais, está a maior eficiência na

FLORESTA, Curitiba, PR, v. 45, n. 4, p. 797 - 808, out. / dez. 2015.

Wojciechowski, J. C. et al.

ISSN eletrônico 1982-4688 / ISSN impresso 0015-3826

DOI: $10.5380 /$ rf.v45i4.37289 
estimativa de parâmetros e avalição da incerteza envolvida na estrutura da dependência, que pode ser facilmente integrada na previsão, ao contrário da geoestatística baseada em variograma (DIGGLE; RIBEIRO JUNIOR, 2007). A máxima verossimilhança é uma ferramenta de grande uso para inferência espacial, incluindo avaliação de significância estatística, cálculo de intervalos de confiança (IC), modelo de avaliação e predição (PLONER; DUTTER, 2000).

O estimador de máxima verossimilhança possui propriedades assintóticas tal como sendo normalmente distribuído, sem viés e eficiente, ao mesmo tempo em que possui flexibilidade para as diferentes transformações da variável resposta, sendo útil para modelagem estatística em geral e para aplicações em geoestatística (DIGGLE; RIBEIRO JUNIOR, 2007)

Segundo Millar (1999) o logaritmo da função da verossimilhança (L-V) é mais utilizado na inferência do que a própria função da verossimilhança, devido a qualidades decorrentes do fato que os algoritmos numéricos possuem melhor comportamento para maximizar a log-verossimilhança do que diretamente a função de verossimilhança. Os valores da probabilidade podem ser pequenos na maioria dos casos, mas também podem ser números extremamente grandes, dependendo do modelo testado e do número de dados para estimar a função, gerando cálculos potencialmente imprecisos pela máxima verossimilhança em relação ao uso da função log-verossimilhança. A curvatura do logaritmo da verossimilhança altera em sua inclinação, conforme a derivada de $2^{\mathrm{a}}$ ordem tendo fundamental importância, tanto na teoria quanto na prática da inferência estatística, pois marca onde o valor é máximo para a função.

Modelos combinados mistos podem ser utilizados para as seguintes finalidades (DEMIDENKO, 2013): Modelar dados com múltiplas fontes de variação; modelar dados agrupados ou longitudinais complexos (dados geográficos); modelar variabilidade e heterogeneidade biológica; como um modelo estatístico para log-verossimilhança; para fornecer uma base teórica fidedigna para o critério de informação de Akaike (AIC); para lidar com parâmetros de multidimensionalidade (reconstituição e calibração de imagens geoposicionadas), dentre outras aplicações matemáticas.

As variáveis dendrométricas como a altura, diâmetro, área basal, biomassa aérea e volume podem demonstrar certa dependência espacial, resultante dos processos naturais de crescimento inerentes à competição por espaços e recurso disponíveis em um determinado local. Essa interdependência pode ser analisada através da distância entre as árvores ou fatores relacionados à estabilização das espécies ao longo do desenvolvimento de uma determinada floresta (WHITMORE, 1990; RODE et al., 2010).

No presente estudo, objetivou-se verificar a dependência espacial e distribuição do volume por hectare em três fragmentos de Floresta Estacional Decidual com uso de um modelo geoestatístico capaz de expressar as diferenças ou semelhanças biológicas desses fragmentos considerando nas análises a combinação dos dados das três áreas.

\section{MATERIAL E MÉTODOS}

\section{Aspectos gerais e fitofisionômicos}

Os fragmentos localizam-se próximos ao município de Santa Tereza, região nordeste do estado do Rio Grande do Sul. As áreas somam 9 hectares subdivididos por critérios de homogeneidade fisionômica e idade pós-intervenção (corte raso de árvores de interesse comercial) em: floresta madura (FMA) com 75 anos; floresta em estágio secundário (FES), com 55 anos e floresta em estágio inicial (FEI) com 30 anos (VACCARO, 1997). A distância entre as áreas FEI e FES é de 0,8 km, enquanto que a área mais afastada (FMA) dista 3,5 km em relação aos outros dois fragmentos. O Clima é classificado como tipo Cfa, subtropical úmido, segundo Köppen (MORENO, 1961).

A vegetação é classificada como Floresta Estacional Decidual $(70 \%$ a $90 \%$ das árvores emergentes perdem completamente as folhas durante o inverno) e pertence a região fitogeográfica da Bacia do rio Jacuí (REITZ et al., 1988). Em relação às fitofisionomias e às espécies encontradas pode-se afirmar que a FMA, apresentou três estratos arbóreos bem definidos e um estrato superior emergente com alguns indivíduos de grande porte, sendo: Gymnanthes concolor Spreng., Nectandra megapotamica (Spreng.) Mez., Sorocea bonplandii (Baill.) W. C. Burger et al. e Trichilha elegans A. Juss., as espécies mais frequentes encontradas na área. Notou-se na FES a existência de dois estratos de porte arbóreos contínuos e bem definidos e um terceiro estrato acima destes em formação, com árvores esparsas e sem entrelaçamento de copas, enquanto que a FEI apresentou dois estratos arbóreos não muito definidos, com um estrato arbustivo denso composto de Cupania vernalis Cambess., Trichilha elegans A. Juss., 
Allophylus edulis (A. St.-Hil. et al.) Radlk. e Luehea divaricata Mart. et Zucc., e um herbáceo composto principalmente de gramíneas para ambas as áreas de FEI e FES.

\section{Amostragem e obtenção dos dados}

Foi utilizada uma amostragem do tipo sistemática onde cada nó da malha de pontos era alocado uma unidade amostral, totalizando 56 parcelas distribuídas de forma a cobrir toda a extensão dos fragmentos. As unidades amostrais (u.a) foram instaladas com distâncias regulares de 40 x $40 \mathrm{~m}$ com auxílio de um mapeamento prévio utilizando GPS e trena, sendo divididas entre as áreas da seguinte forma: 17 na FEI, 20 na FES e 19 na FMA. O efeito de borda foi atenuado tomando-se uma distância mínima de 15 metros para o interior dos fragmentos.

Adotou-se parcela de área variável com o método de amostragem com inclusão das árvores proporcional à distância. A intensidade amostral mínima admitida foi de 10\%, que resultou em parcelas com tamanhos entre 200 a $250 \mathrm{~m}^{2}$, conforme metodologia descrita por Prodan (1965). No entanto, foi adotado como raio da unidade amostral a distância da oitava árvore, ou seja, inclusão de 2 árvores a mais do método de 6 árvores, o que resultou em uma diminuição de $20 \%$ do total da variância por u.a. Assim, foram medidas 8 árvores próximas do ponto amostral de cada parcela, sendo a $8^{\mathrm{a}}$ árvore contada como meia. Todas as árvores com DAP $\geq 10 \mathrm{~cm}$ foram identificadas e medidas suas distâncias, altura comercial e total, para posterior cálculo do volume. Para o cálculo do volume individual comercial utilizou-se um fator de forma " $\mathrm{f}=0,65$ " adotado para florestas deciduais na região serrana do estado do Rio Grande do Sul (UNIVERSIDADE FEDERAL DE SANTA MARIA (UFSM), 2001). A figura 1 ilustra a posição dos fragmentos no estado e em detalhe no município.

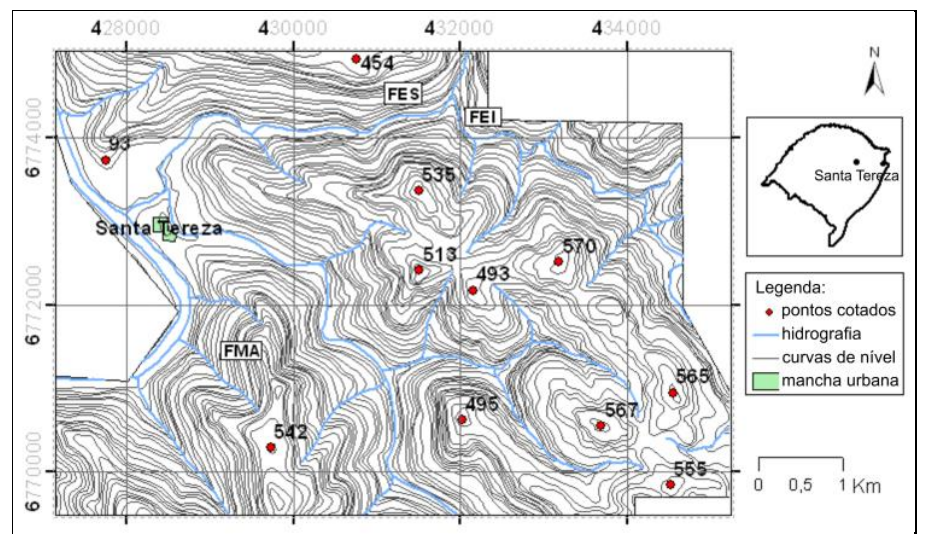

Figura 1. Localização dos fragmentos FEI, FES e FMA próximo ao município de Santa Tereza, RS. Figure 1. Location of fragments FEI, FES and FMA near Santa Tereza, RS municipality.

\section{Análise dos dados}

Foi elaborado a partir da estatística espacial descritiva um gráfico dos quantis, bem como, uma análise que verifica possíveis tendências na distribuição espacial em relação aos eixos de coordenadas X e Y (UTM) e por fim, um histograma de frequência para o volume $\left(\mathrm{m}^{3} \cdot \mathrm{ha}^{-1}\right)$. A análise da transformação dos dados foi realizada pelo método de Box-Cox (BOX; COX, 1964).

\section{Ajuste e seleção dos modelos pela função log-verossimilhança}

Foram testados modelos, individuais M01 (FEI), M02 (FES) e M03 (FMA) e combinados (M04 a M11). Os modelos combinados foram construídos com base na complexidade do número de parâmetros (Npars) resultante da combinação entre $\left(\beta, \tau^{2}, \sigma^{2}\right.$ e $\left.\Phi\right)$, a partir do modelo mínimo (todas as áreas são semelhantes), até o modelo máximo (todas as áreas são distintas) para a variável volume, sendo:

- M04 - Combinação dos parâmetros $\beta, \tau^{2}, \sigma^{2}$ e $\Phi$ iguais nas áreas FEI, FES e FMA (Npars = 4);

- M05 - Combinação da variância espacial $\left(\sigma^{2}\right)$ e o alcance $(\Phi)$ iguais FEI, FES e FMA (Npars =7);

- M06 - Combinação dos parâmetros $\beta, \tau^{2}, \sigma^{2}$ e $\Phi$ iguais nas áreas FEI e FES (Npars = 8);

- M07 - Combinação da média dos volumes $(\beta)$ e efeito pepita $\left(\tau^{2}\right)$ iguais nas áreas FEI e FES, e do alcance $(\Phi)$ na FES e FMA (Npars $=9)$; 
- M08 - Combinação da média dos volumes $(\beta)$ nas áreas FEI e FES e da variância espacial $\left(\sigma^{2}\right)$ e alcance $(\Phi)$ na FES e FMA (Npars $=9)$;

- M09 - Combinação da média $(\beta)$, efeito pepita $\left(\tau^{2}\right)$ e alcance $(\Phi)$ na FEI e FES (Npars = 9);

- M10 - Combinação da variância espacial $\left(\sigma^{2}\right)$ na FEI, FES e FMA (Npars $\left.=10\right)$;

- M11 - Combinação todos os parâmetros $\beta, \tau^{2}, \sigma^{2}$ e $\Phi$ diferente na FEI, FES e FMA (Npars = 12).

Foi utilizado o modelo exponencial para explicar a correlação do $(\log )$ volume nas áreas. Este modelo é um caso particular da função Matèrn (MATÈRN, 1986), que se aproxima do modelo exponencial quando utilizado kappa (к) = 0,5 como parâmetro de suavização (1).

$$
\rho(\mathrm{u})=\left\{2^{\mathrm{K}-1} \Gamma(\mathrm{K})\right\}^{-1}(\mathrm{u} / \Phi)^{\mathrm{K}} \mathrm{K}_{\mathrm{K}}(\mathrm{u} / \Phi)
$$

Foi utilizado o modelo " $\mathrm{yi}=\mathrm{D} \beta+\mathrm{s}\left(\mathrm{x}_{\mathrm{i}}\right)+\mathrm{e}_{\mathrm{i}}$ " como base para o ajuste dos $(\log )$ volumes por verossimilhança, sendo as observações $y_{i}=y_{1}, y_{2}, \ldots, y_{n}$, normalmente distribuídas, em que yi é o vetor da variável resposta observada na posição $\mathrm{x}_{\mathrm{i}}$, $\mathrm{D}$ é uma matriz de covariáveis com dimensão $\mathrm{n} \mathrm{x} ; \beta$ o vetor dos parâmetros da regressão e; $\mathrm{e}_{\mathrm{i}}$, um termo independente associado ao erro aleatório com média " 0 " e

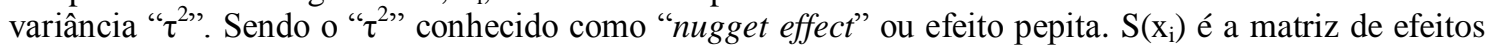
aleatórios na qual admite-se que segue uma distribuição normal multivariada ( $N M V)$ por aproximação dada por $\mathrm{S}\left(\mathrm{x}_{\mathrm{i}}\right) \sim N M V\left[0, \sigma^{2} R(\Phi)\right]$, com média zero; variância $\sigma^{2}$ e função de correlação $\rho$ (u; $\left.\Phi, \mathrm{K}\right)$. Sendo "u" a distância euclidiana entre os pontos observados, em função do grau de associação espacial $\Phi$, do parâmetro de suavização kappa e da matriz de covariâncias $R(\Phi)$ do elemento $\rho$ (u) (DIGGLE; RIBEIRO JUNIOR, 2007).

Dessa forma, os parâmetros do modelo geoestatístico foram estimados pela função do logaritmo da verossimilhança maximizada de acordo com a expressão (2):

$$
L\left(\beta, \tau^{2}, \sigma^{2}, \Phi\right)=-1 / 2\left\{\mathrm{n} \log (2 \mu)+\log \left\{\left|\left(\sigma^{2} R(\Phi)+\tau^{2} \mathrm{I}\right)\right|\right\}+(\mathrm{y}-\mathrm{D} \beta)^{\mathrm{T}}\left(\sigma^{2} R(\Phi)+\tau^{2} \mathrm{I}\right)^{-1}(\mathrm{y}-\mathrm{D} \beta)\right\}
$$

Adotou-se como critério de seleção de modelos a Informação de Akaike (AKAIKE, 1974), para as diferentes relações de covariância dos parâmetros combinados, sendo que o melhor modelo é aquele que apresenta o menor valor de AIC, calculado pela seguinte expressão (3):

$$
\mathrm{AIC}=-2 *(\mathrm{~L}-\mathrm{V})+k * \mathrm{Npar}
$$

em que: $\mathrm{L}-\mathrm{V}=$ logaritmo da verossimilhança maximizada; $k=$ termo que penaliza o modelo com mais parâmetros sendo igual a 2 na fórmula clássica do AIC; Npar = número de parâmetros estimados pelo modelo.

O termo (L-V) foi utilizado para avaliar o comportamento da função frente à transformação logarítmica do conjunto de dados da variável resposta, no caso o volume $\mathrm{em}^{3}$ por hectare. Os dados foram tabulados e analisados utilizando o programa computacional R Core Team (2013), e o pacote geoR (RIBEIRO JUNIOR; DIGGLE, 2001).

\section{RESULTADOS E DISCUSSÃO}

\section{Estatística descritiva e análise exploratória}

Observou-se que na área de FEI, os valores mínimos e máximos da distribuição dos volumes foram, respectivamente, $26,3 \mathrm{~m}^{3}$ e $125,0 \mathrm{~m}^{3}$, com média $56,8 \mathrm{~m}^{3}$ por hectare, divididos em quartis de 40,$2 ; 50,2$ e $66,6 \mathrm{~m}^{3}$. Na FES, os valores mínimos e máximos da distribuição foram $25,1 \mathrm{~m}^{3}$ e $249,0 \mathrm{~m}^{3}$ respectivamente, com média de $90,3 \mathrm{~m}^{3}$ por hectare, com quartis de 42,$4 ; 73,1 \mathrm{e} 111 \mathrm{~m}^{3}$ por hectare.

A FMA obteve valores mínimos e máximos de $10,8 \mathrm{~m}^{3}$ e $265 \mathrm{~m}^{3}$ respectivamente, com média igual a $82,0 \mathrm{~m}^{3}$ por hectare, com quartis de 37,$8 ; 66,1 \mathrm{e} 115,1 \mathrm{~m}^{3}$. No total dos fragmentos, os volumes estimados obtiveram valores de coeficiente de variação máximo de $30 \%$.

A figura 2 apresenta os gráficos de análise exploratória após a retirada dos valores atípicos, para os dados da área FEI. No gráfico "a" estão representados os símbolos dos quartis de uma distribuição empírica dos valores de volume $\left(\mathrm{m}^{3} \mathrm{ha}^{-1}\right)$.

No gráfico "b" e "c" (Figura 2) podemos notar a dispersão linear em razão das coordenadas "x" e "y", indicando uma possível não estacionaridade da média, porém de difícil confirmação com esta 
quantidade de dados. O gráfico "d" mostra um histograma onde observa-se um maior número de indivíduos na classe que compreende os volumes entre 40,0 a $60,0 \mathrm{~m}^{3} \cdot \mathrm{ha}^{-1}$.
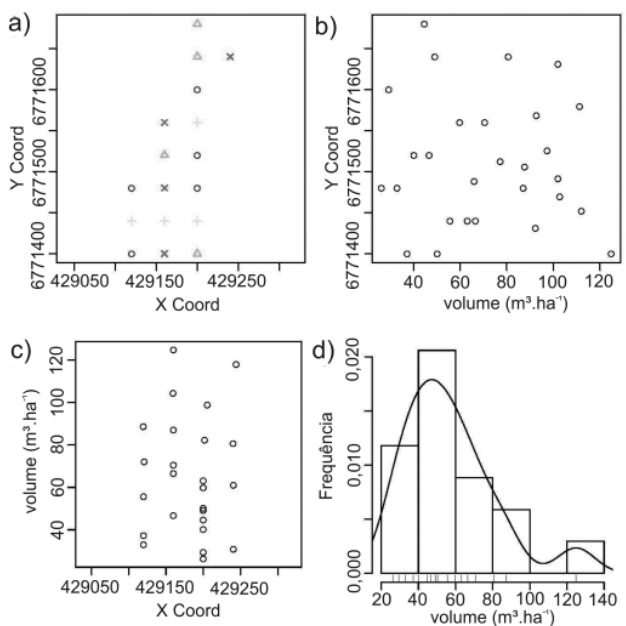

Figura 2. a) Parcelas (+ = valor mínimo até $25 \%$ do total observado, $\Delta=25$ a $50 \%, \mathrm{o}=50$ a $75 \%$ e $\times=$ $75 \%$ até o valor máximo observado); b) e c) dispersão dos valores contra as coordenadas da área; d) histograma de frequência do volume $\left(\mathrm{m}^{3} \cdot \mathrm{ha}^{-1}\right)$ na área de FEI.

Figure 2. a) Samples ( $+=$ minimum value up to $25 \%$ of total observed, $\Delta=25$ to $50 \%, o=50$ to $75 \%$ and $\times=75 \%$ to maximum value observed); b) and c) dispersion values from area coordinates; d) volume frequency histogram $\left(\mathrm{m}^{3} \cdot \mathrm{ha}^{-1}\right)$ in FEI area.

Analisando o conjunto de informações para a FES e FMA (Figuras 3 e 4), a dispersão dos dados contra as coordenadas " $x$ " e " $y$ " parecem semelhantes para ambas as áreas, sem tendências evidentes em nenhum sentido do eixo de coordenadas, porém, mal distribuídos. Tal fato pode gerar variogramas transitivos (sem partamar), dificultando a aplicação dos ajustes por esse método (VIEIRA, 1995).
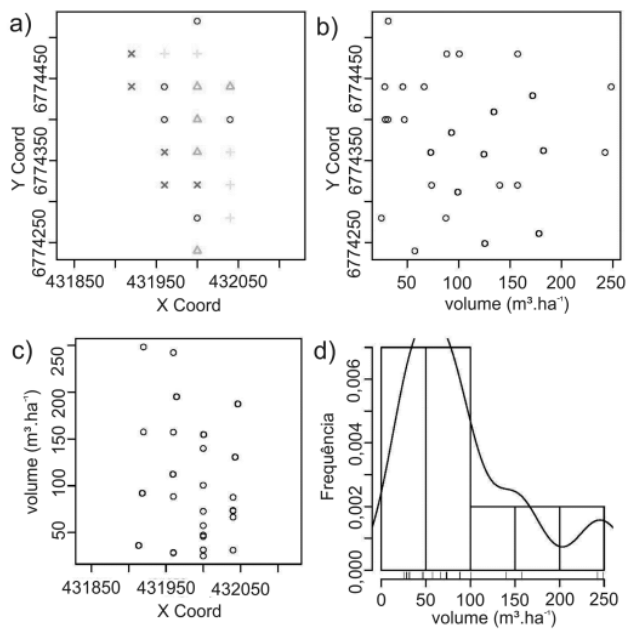

Figura 3. a) Parcelas (+ = valor mínimo até $25 \%$ do total observado, $\Delta=25$ até $50 \%, o=50$ até $75 \%$ do total observado, $x=75 \%$ ao valor máximo observado); b) e c) dispersão dos valores contra as coordenadas da área; d) histograma de frequência do volume $\left(\mathrm{m}^{3} \cdot \mathrm{ha}^{-1}\right)$ na área de FES.

Figure 3. a) Samples ( $+=$ minimum value up to $25 \%$ of total observed, $\Delta=25$ to $50 \%, o=50$ to $75 \%, \times$ $=75 \%$ to maximum value observed); b) and c) dispersion values from area coordinates; $d$ ) volume frequency histogram $\left(\mathrm{m}^{3} \cdot \mathrm{ha}^{-1}\right)$ in FES area. 

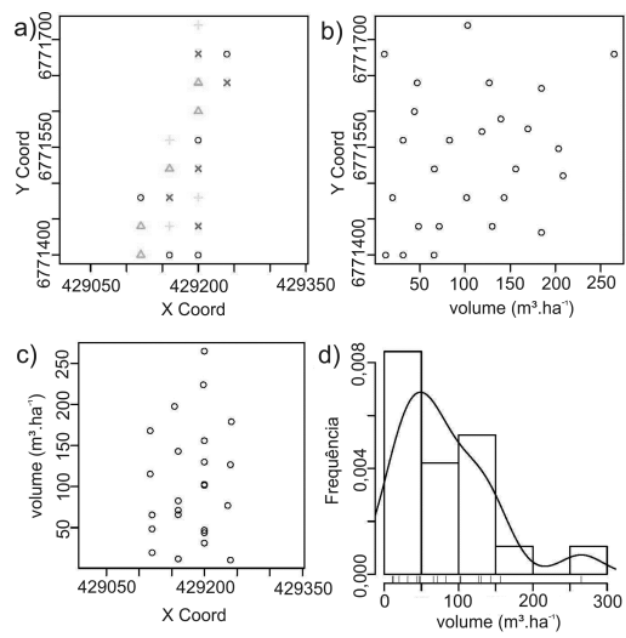

Figura 4. a) Parcelas (+= valor mínimo até $25 \%$ do total observado, $\Delta=25$ até $50 \%, o=50$ até $75 \%, x=$ $75 \%$ ao valor máximo observado); b) e c) dispersão dos valores contra as coordenadas da área; d) histograma de frequência do volume $\left(\mathrm{m}^{3} \cdot \mathrm{ha}^{-1}\right)$ na área de FMA.

Figure 4. a) Samples ( $+=$ minimum value up to $25 \%$ of total observed, $\Delta=25$ to $50 \%, o=50$ to $75 \%, x$ $=75 \%$ to maximum value observed); b) and c) dispersion values from area coordinates; $\mathrm{d}$ ) volume frequency histogram $\left(\mathrm{m}^{3} \cdot \mathrm{ha}^{-1}\right)$ in FMA area.

O histograma na FES (Figura 3), revela maior concentração dos volumes entre o intervalo de 0 a $100 \mathrm{~m}^{3} \cdot \mathrm{ha}^{-1}$, enquanto na FMA, entre os intervalos de 0 a 50 e de 100 a $150 \mathrm{~m}^{3} \cdot \mathrm{ha}^{-1}$, mostrando falhas na distribuição, oriundas de classes do volume faltantes, padrões típicos de florestas que sofreram cortes seletivos de indivíduos de maior interesse comercial (SCHNEIDER; FINGER, 2000).

Os gráficos de histogramas nas figuras 2,3 e 4, bem como os testes de normalidade, indicaram a necessidade da transformação dos dados de volume $\left(\mathrm{m}^{3} \cdot \mathrm{ha}^{-1}\right)$, sendo a normalidade dos dados é uma exigência na aplicação das técnicas de modelagem pelo método de máxima verossimilhança, no qual tem por base a distribuição de Gauss (DIGGLE; RIBEIRO JUNIOR, 2007).

Em análise conjunta do perfil da verossimilhança de Box-Cox, o teste resultou em um lambda $(\lambda)$ igual a 0 , que corresponde a transformação logarítmica. $O$ resultado comum para as três áreas possibilitou a integração dos modelos combinando a informação dos volumes.

Para Vuolo (1996) a transformação obtida melhora a aproximação à normalidade, porém é necessária cautela, pois, não há garantias de que mesmo a melhor escolha de transformação a ser realizada produzirá um conjunto de dados transformados que seja adequado à suposição de normalidade.

E possível após uma transformação, alguns dos valores originalmente fora do conjunto de dados podem acompanhar a distribuição (KITANIDIS, 1997). O autor ressalta ainda que, os resultados obtidos por uma transformação devem ser cuidadosamente examinados, pois em muitos casos, os chamados valores extremos são as medidas mais interessantes no conjunto de dados.

Essa recomendação de fato vale para qualquer transformação usada em modelos. A transformação reduziu a variância do volume, que no caso dos dados transformados, acompanham a média.

\section{Ajuste dos modelos pela função log-verossimilhança}

$\mathrm{Na}$ tabela 1 se encontram os resultados do ajuste para as áreas em separado, onde o modelo "M02" obteve um alcance prático (a) de 44,03 m, enquanto que os modelos "M01" e "M03" para o mesmo parâmetro foram 7,43 m e 7,62 m respectivamente.

Os valores do ajuste indicam ser possível, dado o espaçamento da malha adotada nas parcelas de $40 \mathrm{~m}$, explicar a estrutura da continuidade espacial de maneira satisfatória apenas no fragmento FES onde $o$ alcance foi maior.

Assim sendo, a estimativa individual das áreas não acarretaria em vantagens do ponto de vista da predição do fenômeno estudado, pois teríamos apenas uma superfície com a distribuição dos volumes modelada pelo respectivo valor médio. 
Tabela 1. Estimativa dos parâmetros ajustados por log-verossimilhança aplicada à variável volume nas áreas FEI-FES-FMA.

Table 1. Estimation of parameters adjusted by log-likelihood applied to volume variable in FEI-FESFMA areas.

\begin{tabular}{|c|c|c|c|c|c|c|}
\hline \multirow{2}{*}{ Modelos } & \multicolumn{5}{|c|}{ Estimativas (4 parâmetros) } & \multirow{2}{*}{$\mathbf{a}$} \\
\hline & $\beta$ & $\tau^{2}$ & $\sigma^{2}$ & $\tau^{2}+\sigma^{2}$ & $\Phi$ & \\
\hline M01 (FEI) & 3,995 & 0,151 & 0,005 & 0,156 & 2,4747 & 7,43 \\
\hline M02 (FES) & 4,273 & 0,001 & 0,474 & 0,475 & 14,697 & 44,03 \\
\hline M03 (FMA) & 4,101 & 0,685 & 0,025 & 0,710 & 2,546 & 7,62 \\
\hline
\end{tabular}

\section{Ajuste dos modelos pela função log-verossimilhança combinada}

Observa-se na tabela 2 o ajuste do modelo (M04) mais parcimonioso ou modelo mínimo, partindo da suposição de que todas as áreas são semelhantes, ou seja, possuem valores muito próximos de seus volumes médios $(\beta)$, variância não espacial $\left(\tau^{2}\right)$ bem como os componentes pertencentes à variabilidade espacial explicada $\left(\sigma^{2}\right)$ e o alcance $(\Phi)$.

Em uma análise preliminar, partindo da premissa básica que os fragmentos pertencem à mesma fitofisionomia e que diferem somente em idades pós-intervenção, a resposta do grau de associação espacial entre as parcelas para o modelo resultou em um o alcance prático abaixo do espaçamento entre as amostras $(\mathrm{a}=9,15 \mathrm{~m})$. Dessa forma, têm-se uma variabilidade explicada até essa distância.

Outro ponto a ser observado é a relação sinal-ruído que é a razão da variância espacial pela variância aleatória, considerada fraca para esse modelo (BONAT et al., 2011).

Tabela 2. Estimativa dos parâmetros ajustados por log-verossimilhança aplicada à variável volume combinando $\left(\beta, \tau^{2}, \sigma^{2}\right.$ e $\left.\Phi\right)$ do modelo nas áreas FEI-FES-FMA.

Table 2. Estimation of parameters adjusted by log-likelihood applied to volume variable combining ( $\beta$, $\tau^{2}, \sigma^{2}$ and $\left.\Phi\right)$ of model in FEI-FES-FMA areas.

\begin{tabular}{|c|c|c|c|c|c|c|}
\hline \multirow{2}{*}{ M04 } & \multicolumn{5}{|c|}{ Estimativas (4 parâmetros) } & \multirow[b]{2}{*}{$\mathbf{a}$} \\
\hline & $\beta$ & $\overline{\tau^{2}}$ & $\sigma^{2}$ & $\tau^{2}+\sigma^{2}$ & $\Phi$ & \\
\hline $\begin{array}{l}\text { FEI } \\
\text { FES } \\
\text { FMA }\end{array}$ & 4,1168 & 0,0070 & 0,4939 & 0,5009 & 3,0493 & 9,15 \\
\hline
\end{tabular}

Na tabela 3 são apresentados os resultados de outro novo modelo (M05) que testa a possibilidade das áreas possuírem o mesmo componente estrutural, considerando comuns a variabilidade espacial $\left(\sigma^{2}\right)$ e o alcance $(\Phi)$. Esse modelo estimou 8 parâmetros sendo elaborado de modo a ajustar valores diferentes para média do volume $(\beta)$ e variabilidade não espacial $\left(\tau^{2}\right)$ em cada área.

Tabela 3. Estimativa dos parâmetros ajustados por log-verossimilhança aplicada à variável volume combinando $\left(\sigma^{2}\right.$ e $\left.\Phi\right)$ do modelo nas áreas FEI-FES-FMA.

Table 3. Estimation of parameters adjusted by log-likelihood applied to volume variable combining $\left(\sigma^{2}\right.$ and $\Phi)$ of model in FEI-FES-FMA areas.

\begin{tabular}{|c|c|c|c|c|c|c|}
\hline \multirow{2}{*}{ M05 } & \multicolumn{5}{|c|}{ Estimativas (8 parâmetros) } & \multirow[b]{2}{*}{$\mathbf{a}$} \\
\hline & $\boldsymbol{\beta}$ & $\tau^{2}$ & $\sigma^{2}$ & $\tau^{2}+\sigma^{2}$ & $\Phi$ & \\
\hline FEI & 3,9599 & 0,0020 & \multirow{3}{*}{0,3206} & 0,3226 & \multirow{3}{*}{5,2162} & \multirow{3}{*}{15,65} \\
\hline FES & 4,2648 & 0,0020 & & 0,3226 & & \\
\hline FMA & 4,1014 & 0,3838 & & 0,7044 & & \\
\hline
\end{tabular}

$\beta$ : média dos volumes; $\tau^{2}$ : efeito pepita; $\sigma^{2}:$ contribuição; $\tau^{2}+\sigma^{2}$ : patamar; $\Phi$ : alcance; a: alcance prático $(3 \Phi$ para kappa 0,5$)$.

Analisando os resultados das estimativas dos parâmetros para esse cenário, houve uma alteração no alcance (a) de 9,15 m no modelo "M04" para 15,56 m no modelo "M05". Contudo não se configura em um modelo adequado, pois o alcance prático de $15,65 \mathrm{~m}$ ainda é inferior ao espaçamento mínimo entre parcelas.

Os modelos combinados "M04" e "M05" resultaram em uma perda do grau de dependência espacial em relação ao encontrado no modelo ajustado para as áreas individuais "M02". Sendo que, o

FLORESTA, Curitiba, PR, v. 45, n. 4, p. 797 - 808, out. / dez. 2015.

Wojciechowski, J. C. et al.

ISSN eletrônico 1982-4688 / ISSN impresso 0015-3826

DOI: 10.5380/rf.v45i4.37289 
alcance do modelo foi reduzido de 44,03 m para 15,65 e 9,15 m respectivamente nos modelos "M05" e "M04". Dessa forma, as áreas podem tanto combinar informações com a finalidade de explicar a dependência espacial da variável resposta, como também, a resultante dessa combinação, pode reduzir a extensão da correlação espacial de acordo com a configuração do modelo.

Wu (2010) comenta que, em se tratando de modelos mistos a combinação resultante da tentativa de ajuste dos parâmetros, tem inferência direta na matriz de variância e covariância dos modelos. Isso é possível, pois os modelos foram construídos de forma a garantir que as áreas analisadas expressem suas similaridades ou diferenças, dando a mesma chance para ambas ocorrerem.

O modelo "M06" relaciona duas das áreas que são mais próximas, no caso a áreas FEI e FES $(0,8 \mathrm{~km})$ em relação à área FMA $(3,5 \mathrm{~km})$, combinando os parâmetros $\left(\beta, \tau^{2}, \sigma^{2} \mathrm{e} \Phi\right)$ entre as áreas FEI e FES, com separabilidade na área de floresta madura (FMA), conforme tabela 4.

Tabela 4. Estimativa dos parâmetros ajustados por log-verossimilhança aplicada à variável volume com combinação dos parâmetros $(\beta, \tau 2, \sigma 2$ e $\Phi)$ do modelo nas áreas FEI-FES.

Table 4. Estimation of parameters adjusted by log-likelihood applied to volume with combination of parameters $\left(\beta, \tau^{2}, \sigma^{2}\right.$ e $\left.\Phi\right)$ of model in FEI-FES areas.

\begin{tabular}{|c|c|c|c|c|c|c|}
\hline \multirow[b]{2}{*}{ M06 } & \multicolumn{5}{|c|}{ Estimativas ( 8 parâmetros) } & \multirow[b]{2}{*}{$\mathbf{a}$} \\
\hline & $\boldsymbol{\beta}$ & $\tau^{2}$ & $\sigma^{2}$ & $\tau^{2}+\sigma^{2}$ & $\Phi$ & \\
\hline $\begin{array}{l}\text { FEI } \\
\text { FES }\end{array}$ & 4,1247 & 0,0069 & 0,3439 & 0,3508 & 13,1749 & 39,52 \\
\hline FMA & 4,1014 & 0,2039 & 0,6892 & 0,8931 & 3,4366 & 10,31 \\
\hline
\end{tabular}

Pode-se observar no parâmetro alcance $(\Phi)$, que houve um aumento da extensão da correlação espacial em relação aos modelos anteriores $(15,65 \mathrm{~m}$ para $39,52 \mathrm{~m})$, porém ainda abaixo do menor espaçamento entre os pontos. Dessa forma temos que, as áreas FEI e FES, em conjunto, nessa configuração do modelo, não explicam a variabilidade dos volumes em toda a extensão das mesmas.

Ao truncar os parâmetros do modelo para determinada hipótese incluímos uma condição, ou inferência a priori para analisar seus efeitos no modelo a posteriori. O resultado do processo é analisado de forma integrada, porém, antes dos testes de validação, um parâmetro importante a ser observado é o alcance prático (a), pois representa uma distância em metros com seu resultado obtido pela multiplicação do valor do parâmetro $(\Phi)$ por $3(k a p p a=0,5)$ para função de correlação exponencial.

Os parâmetros alcance $(\Phi)$ e suavidade kappa $(k)$ do modelo Matèrn não são ortogonais, isso implica que as escalas correspondentes as diferentes ordens de correlação Matèrn não são diretamente comparáveis, mas uma depende da outra. Para tal, suas aproximações são dadas para o alcance prático em $3 ; 4,75$ e 5,9 $\Phi$, para o parâmetro kappa igual a 0,$5 ; 1,5$ e 2,5 respectivamente, e $\sqrt{ } 3 \Phi$ para função de correlação gaussiana (DIGGLE; RIBEIRO JUNIOR, 2007)

Foi testado um modelo intermediário com 9 parâmetros ajustados "M07" (Tabela 5), combinando a média $(\beta)$ e o nugget $\left(\tau^{2}\right)$ para as áreas FEI e FES, e fixando o alcance $(\Phi)$ do modelo nas áreas de FES e FMA. Nessa condição, os padrões espaciais para o volume possuem forte correlação de continuidade espacial nas áreas quando combinando a informação desses dois fragmentos, corroborado pelos valores do alcance prático que aumentaram de 39,52 m (M06) para 66,09 m nas áreas.

Tabela 5. Estimativa dos parâmetros ajustados por log-verossimilhança aplicada à variável volume combinando $\left(\beta\right.$ e $\left.\tau^{2}\right)$ do modelo nas áreas FEI-FES e $(\Phi)$ nas áreas FES-FMA.

Table 5. Estimation of parameters adjusted by log-likelihood applied to volume variable combining ( $\beta$ and $\tau^{2}$ ) of model in FEI-FES areas and $(\Phi)$ in FES-FMA areas.

\begin{tabular}{|c|c|c|c|c|c|c|}
\hline \multirow{2}{*}{ M07 } & \multicolumn{5}{|c|}{ Estimativas (9 parâmetros) } & \multirow[b]{2}{*}{$\mathbf{a}$} \\
\hline & $\beta$ & $\tau^{2}$ & $\sigma^{2}$ & $\tau^{2}+\sigma^{2}$ & $\Phi$ & \\
\hline FEI & \multirow{2}{*}{4,0155} & \multirow{2}{*}{0,0007} & 0,1585 & 0,1592 & 0,5043 & 1,51 \\
\hline FES & & & 0,5374 & 0,5381 & \multirow{2}{*}{22,0324} & 66,09 \\
\hline FMA & 4,1014 & 0,7097 & 0,1211 & 0,7098 & & 66,09 \\
\hline
\end{tabular}

Na tabela 6 são apresentados os resultados dos testes com hipótese de que o volume médio $(\beta)$ e semelhante nos fragmentos FEI e FES, com a variabilidade associada ao erro aleatório $\left(\tau^{2}\right)$ diferente entre as áreas, porém como os componentes da variabilidade espacial estruturada $\sigma^{2}$ e $\Phi$ semelhante para FES e FMA. 
O modelo obteve um alcance prático igual a 35,37 m para as áreas FES e FMA. Na floresta em estágio inicial (FEI) o alcance prático (7,62 m) ficou bem abaixo do menor espaçamento entre as parcelas (40 m).

Tabela 6. Estimativa dos parâmetros ajustados por log-verossimilhança aplicada à variável volume combinando $(\beta)$ do modelo nas áreas FEI-FES e $\left(\sigma^{2}\right.$ e $\left.\Phi\right)$ nas áreas FES-FMA.

Table 6. Estimation of parameters adjusted by log-likelihood applied to volume variable combining ( $\beta$ ) of model in FEI-FES areas and $\left(\sigma^{2}\right.$ and $\left.\Phi\right)$ in FES-FMA areas.

\begin{tabular}{|c|c|c|c|c|c|c|}
\hline \multirow{2}{*}{ M08 } & \multicolumn{5}{|c|}{ Estimativas (9 parâmetros) } & \multirow[b]{2}{*}{$\mathbf{a}$} \\
\hline & $\beta$ & $\tau^{2}$ & $\sigma^{2}$ & $\tau^{2}+\sigma^{2}$ & $\Phi$ & \\
\hline FEI & \multirow{2}{*}{4,0394} & 0,0195 & 0,1426 & 0,1620 & 2,5388 & 7,62 \\
\hline FES & & 0,0070 & \multirow{2}{*}{0,5084} & 0,5154 & \multirow{2}{*}{11,7897} & 35,37 \\
\hline FMA & 4,0980 & 0,2169 & & 0,7253 & & 35,37 \\
\hline
\end{tabular}

O modelo "M09" ajustou os parâmetros levando em conta o compartilhamento de $\beta, \tau^{2}$ e $\Phi$ para as áreas FEI-FES, enquanto que o parâmetro da variabilidade espacial $\left(\sigma^{2}\right)$ foi diferente para as 3 áreas. Essa condição ajustou um modelo que é forte candidato a predição em uma primeira análise, visto que o alcance prático foi o maior dentre os modelos testados até o momento $(75,54 \mathrm{~m})$.

Sendo confirmado esse modelo pelos critérios de seleção propostos, a espacialização do volume se daria apenas em 2 áreas (FES e FMA). Percebe-se até agora a ausência de um modelo que explique de maneira satisfatória, através da inferência resultante dos seus parâmetros combinados, a variabilidade espacial em todas as áreas (Tabela 7).

Segundo Pélissier e Goreaud (2001), a formação de padrões em sistemas biológicos é normalmente muito mais complexa, pois possuem como característica forte a heterogeneidade espacial, visto que métodos clássicos de estudos de padrões e distribuição do ponto de vista espacial costumam assumir a hipótese da homogeneidade.

Tabela 7. Estimativa dos parâmetros ajustados por log-verossimilhança aplicada à variável volume combinando $\left(\beta, \tau^{2}\right.$ e $\left.\Phi\right)$ do modelo nas áreas FEI-FES.

Table 7. Estimation of parameters adjusted by log-likelihood applied to volume variable combining ( $\beta$, $\tau^{2}$ and $\left.\Phi\right)$ of model in FEI-FES areas.

\begin{tabular}{|c|c|c|c|c|c|c|}
\hline \multirow{2}{*}{ M09 } & \multicolumn{5}{|c|}{ Estimativas (9 parâmetros) } & \multirow[b]{2}{*}{$\mathbf{a}$} \\
\hline & $\beta$ & $\tau^{2}$ & $\sigma^{2}$ & $\tau^{2}+\sigma^{2}$ & $\Phi$ & \\
\hline FEI & \multirow{2}{*}{4,0154} & \multirow{2}{*}{0,1582} & 0,0700 & 0,2282 & 251811 & 7554 \\
\hline FES & & & 0,4723 & 0,6305 & 25,1811 & 15,34 \\
\hline FMA & 4,1014 & 0,0597 & 0,7055 & 0,7652 & 0,1932 & 0,57 \\
\hline
\end{tabular}

O modelo "M10" supõe que as áreas possuem em comum a distribuição espacial dos volumes $\left(\sigma^{2}\right)$ de seus indivíduos no espaço, diferindo em suas médias $(\beta)$, erro aleatório $\left(\tau^{2}\right)$ e alcance do processo $(\Phi)$. Conforme pode ser observado na tabela 8 , o modelo ajustou os parâmetros para essa condição e obteve um alcance de 3,43 m; 46,69 m e 9,21 m respectivamente para FEI, FES e FMA.

Tabela 8. Estimativa dos parâmetros ajustados por log-verossimilhança aplicada à variável volume combinando $\left(\sigma^{2}\right)$ do modelo nas áreas FEI-FES-FMA.

Table 8. Estimation of parameters set by log-likelihood applied to volume variable combining $\left(\sigma^{2}\right)$ of model in FEI-FES-FMA areas.

\begin{tabular}{|c|c|c|c|c|c|c|}
\hline \multirow[b]{2}{*}{ M10 } & \multicolumn{5}{|c|}{ Estimativas (10 parâmetros) } & \multirow[b]{2}{*}{$\mathbf{a}$} \\
\hline & $\beta$ & $\tau^{2}$ & $\sigma^{2}$ & $\tau^{2}+\sigma^{2}$ & $\Phi$ & \\
\hline FEI & 3,9599 & 0,0009 & \multirow{3}{*}{0,1557} & 0,1562 & 1,1442 & 3,43 \\
\hline FES & 4,2682 & 0,3164 & & 0,04926 & 15,5642 & 46,69 \\
\hline FMA & 4,1014 & 0,5539 & & 0,7153 & 3,0692 & 9,21 \\
\hline
\end{tabular}

Agora, assumiu-se a priori, para ajuste da equação o modelo "M11", que todas as áreas possuem características distintas, ou seja, sem estipular efeitos fixos. Esse modelo considera somente os efeitos

FLORESTA, Curitiba, PR, v. 45, n. 4, p. 797 - 808, out. / dez. 2015.

Wojciechowski, J. C. et al.

ISSN eletrônico 1982-4688 / ISSN impresso 0015-3826

805

DOI: $10.5380 /$ rf.v45i4.37289 
aleatórios, deixando os parâmetros livres para as estimativas das médias $(\beta)$ para os valores observados de volume, variabilidade espacial $\left(\sigma^{2}\right)$, erro aleatório $\left(\tau^{2}\right)$ e grau de dependência espacial (a), distintos para todas as áreas.

Tabela 9. Estimativa dos parâmetros ajustados por log-verossimilhança aplicada à variável volume nas áreas FEI-FES-FMA.

Table 9. Estimation of parameters adjusted by log-likelihood applied to volume variable in FEI-FESFMA areas.

\begin{tabular}{|c|c|c|c|c|c|c|}
\hline \multirow{2}{*}{ M11 } & \multicolumn{5}{|c|}{ Estimativas (12 parâmetros) } & \multirow[b]{2}{*}{$\mathbf{a}$} \\
\hline & $\beta$ & $\tau^{2}$ & $\sigma^{2}$ & $\tau^{2}+\sigma^{2}$ & $\Phi$ & \\
\hline FEI & 3,9600 & 0,0005 & 0,1556 & 0,1561 & 0,2763 & 0,83 \\
\hline FES & 4,2733 & 0,0012 & 0,4722 & 0,4734 & 14,7003 & 44,10 \\
\hline FMA & 4,1014 & 0,0042 & 0,7054 & 0,7096 & 0,2104 & 0,63 \\
\hline
\end{tabular}

Em análise, as áreas mais fracas do ponto de vista do número de parcelas (FEI-FMA) perderam parte do alcance para FES, tendo seu alcance prático aumentado para 44,10 m. Sendo esse modelo, algo mais próximo do ajuste para as áreas individuais $(\mathrm{M} 02, \mathrm{a}=44,03 \mathrm{~m})$, dado a pequena diferença entre os alcances encontrados de um modelo para o outro. Isto indica que os efeitos livres (todas as áreas diferentes) dos parâmetros de um modelo combinado podem ser muito próximos aos ajustes individuais.

\section{Seleção do modelo pelo critério de Akaike (AIC) e alcance (a)}

$\mathrm{Na}$ tabela 11 é apresentado o ranking dos modelos ajustados em ordem crescente de AIC. Observa-se que os modelos ajustados pelo método de combinação das áreas obtiveram sucesso em melhorar as estimativas dos parâmetros, que apresentaram valores de AIC menores em comparação aos modelos ajustados individualmente (M01, M02 e M03). O maior valor do AIC dos modelos compartilhados (M11, AIC = 129,77), é inferior ao menor valor encontrado nos modelos ajustados individualmente (M01, AIC=159,3). A utilização de covariáveis como altura, ou mesmo variáveis de distância, podem melhorar substancialmente a interpretação dos efeitos mistos nos modelos ajustados por máxima verossimilhança, melhorando os valores do AIC (GREGOIRE; SCHABENBERGER, 1994)

Tabela 10. Seleção pelo AIC e alcance prático dos modelos combinados nas áreas FEI, FES, FMA.

Table 10. Selection of combined models in FEI, FES, FMA areas by AIC and practical range.

\begin{tabular}{|c|c|c|c|c|c|c|c|}
\hline \multirow{2}{*}{ Mod. } & \multicolumn{4}{|c|}{ Parâmetros } & \multirow{2}{*}{ L-V } & \multirow{2}{*}{ (a) } & \multirow{2}{*}{ AIC } \\
\hline & $\beta$ & $\tau^{2}$ & $\sigma^{2}$ & $\Phi$ & & & \\
\hline M04 & fei=fes $=$ fma & fei $=\mathrm{fes}=\mathrm{fma}$ & fei $=\mathrm{fes}=\mathrm{fma}$ & fei=fes $=$ fma & $-58,4$ & 9 & 124,99 \\
\hline M07* & $(\mathrm{fei}=\mathrm{fes}) \neq \mathrm{fma}$ & (fei $=$ fes $) \neq$ fma & fei $\neq$ fes $\neq$ fma & fei $\neq(f e s=f m a)$ & $-54,0$ & $2,66,66$ & 125,02 \\
\hline M09 & $(\mathrm{fe} i=\mathrm{fes}) \neq \mathrm{fma}$ & (fei $=$ fes $) \neq$ fma & fei $\neq$ fes $\neq$ fma & $(\mathrm{fei}=\mathrm{fes}) \neq \mathrm{fma}$ & $-54,0$ & $75,75,1$ & 125,11 \\
\hline M05 & fei $\neq$ fes $\neq$ fma & (fei $=$ fes $) \neq$ fma & $\mathrm{fei}=\mathrm{fes}=\mathrm{fma}$ & $\mathrm{fei}=\mathrm{fes}=\mathrm{fma}$ & $-55,5$ & 16 & 125,18 \\
\hline M10 & fei $\neq$ fes $\neq$ fma & fei $\neq$ fes $\neq$ fma & $\mathrm{fei}=\mathrm{fes}=\mathrm{fma}$ & fei $\neq$ fes $\neq$ fma & $-52,9$ & $3,47,9$ & 125,86 \\
\hline M08 & $(\mathrm{fei}=\mathrm{fes}) \neq \mathrm{fma}$ & fei $\neq$ fes $\neq$ fma & fei $\neq(f e s=f m a)$ & fei $\neq(f e s=f m a)$ & $-54,2$ & $8,35,35$ & 126,59 \\
\hline M06 & $(\mathrm{fe} i=\mathrm{fes}) \neq \mathrm{fma}$ & $(\mathrm{fei}=\mathrm{fes}) \neq$ fma & $(\mathrm{fei}=\mathrm{fes}) \neq \mathrm{fma}$ & $(\mathrm{fei}=\mathrm{fes}) \neq \mathrm{fma}$ & $-56,7$ & $39,39,10$ & 129,55 \\
\hline M11 & fei $\neq$ fes $\neq$ fma & fei $\neq$ fes $\neq$ fma & fei $\neq$ fes $\neq$ fma & fei $\neq$ fes $\neq$ fma & $-52,8$ & $1,44,1$ & 129,77 \\
\hline M01 & - & - & - & - & $-75,6$ & 7 & 159,30 \\
\hline M03 & - & - & - & - & $-105,1$ & 7 & 218,30 \\
\hline M02 & - & - & - & - & $-111,9$ & 44 & 231,30 \\
\hline
\end{tabular}

Mod.: modelo matemático; *: melhor modelo pelo critério de Akaike (AIC) e alcance prático (a: $3 \Phi$ para kappa 0,5); $\beta$ : vetor de médias; $\tau^{2}$ : nugget; $\sigma^{2}$ e $\Phi$ : correlação espacial; L-V: log-verossimilhança para FEI, FES, FMA respectivamente.

O modelo "M07" obteve o melhor ajuste dentre os modelos testados com um AIC de 125,02 e extensão da correlação espacial $3 \Phi=66,09 \mathrm{~m}$. Outro ponto que deve ser levado em consideração quando se trata de modelos combinados, é que o modelo deve explicar a dependência espacial da variável em estudo, no maior número de locais possíveis. Quando se tratam de áreas compostas, o parâmetro alcance é um indicativo importante como resultado do processo de escolha, sendo também um dos critérios adotados para predição da superfície utilizando krigagem.

O melhor valor de AIC encontrado foi no modelo "M04", porem o grau de dependência espacial resultante foi de apenas $9 \mathrm{~m}$, indicando que a correlação entre os pontos, na condição de igualdade para as 
três áreas e fraca. Os modelos "M07" e "M09" aparecem como boas alternativas para relação de dependência espacial, com valores de alcance prático de $66 \mathrm{~m}$ (FES-FMA) e $75 \mathrm{~m}$ (FEI-FES), respectivamente e valores de AIC próximos do modelo "M04".

O modelo "M11" ajustado com 12 parâmetros, que testa a hipótese de que a média dos volumes $(\beta)$, o nugget $\left(\tau^{2}\right)$, a variabilidade espacial $\left(\sigma^{2}\right)$ e o alcance $(\Phi)$ são características individuas de cada área, resultou em um valor mais alto de AIC $(129,77)$, além de um alcance $(\Phi)$ igual a $1 \mathrm{~m}$ nas áreas FEI e FMA, e um alcance prático na FES de $44 \mathrm{~m}$. Esse modelo de acordo com os critérios estabelecidos de parcimônia, maximização da função log-verossimilhança e grau de dependência espacial obteve uma relação fraca do ponto de vista da estrutura espacial dos volumes.

Foram selecionados a título de comparação dos parâmetros, os modelos que obtiveram os melhores resultados em relação ao valor do teste de AIC e grau de dependência espacial (maior valor de $\Phi)$, de forma a checar a congruência entre os métodos de ajuste utilizados.

Os modelos "M09" e "M07" combinaram a mesma informação dos parâmetros $\left(\beta, \tau^{2}, \sigma^{2}\right)$, ocorrendo dependência espacial em até $66 \mathrm{~m}$ na FES e FMA no modelo "M07" e até $75 \mathrm{~m}$ no modelo "M09" para as áreas FEI e FES. Esse resultado, do ponto de vista geoestatístico é importante pois, mostra que ocorreu dependência espacial entre duas áreas distantes $3,5 \mathrm{~km}$ entre si, que combinaram seus parâmetros explicando a variabilidade espacial do volume existente entre elas, com uma boa sobreposição do alcance $(\Phi)$ em relação à malha amostral.

\section{CONCLUSÕES}

- Os ajustes dos modelos individuais não foram satisfatórios para explicar a dependência espacial dos volumes por hectare nas áreas.

- O modelo "M07" foi o melhor dentre todos os modelos testados apresentando um AIC de 125,02 e um alcance prático (a) para as áreas de FES e FMA de 66 m.

- Em comparação aos modelos ajustados para as áreas individualmente, os modelos combinados foram superiores para os valores de AIC, grau de dependência espacial e valor máximo de função do logaritmo da verossimilhança (L-V).

- O critério de Akaike (AIC) adotado para seleção dos modelos foi eficiente, uma vez que comparou os diferentes níveis de ajustes propostos na metodologia do trabalho, selecionando um modelo compatível com a realidade das áreas e com os padrões da distribuição espacial do volume.

- É possível recuperar informações de locais com baixa amostragem, através dos modelos combinados, gerando estimativas de volume por hectare distribuído espacialmente.

- Os resultados ressaltam a necessidade de que malhas amostrais de pontos não sejam completamente regulares, impondo um espaçamento mínimo entre as amostras possivelmente abaixo da extensão de dependência espacial. É recomendado que a amostragem contemple uma configuração com pontos amostrais à diferentes distâncias, incluindo pequenas distâncias. Tais configurações são mais úteis para ajustes de modelos espaciais em florestas naturais permitindo uma espacialização adequada.

- O uso de modelos geoestatísticos combinados baseados em verossimilhança para classificação de unidades homogêneas em florestas naturais é uma alternativa promissora em relação aos métodos de ajuste por semivariogramas, pois amplia o poder das análises e torna possível um melhor uso da informação proveniente das diferentes áreas.

\section{AGRADECIMENTOS}

O autor agradece ao Conselho Nacional de Desenvolvimento Científico e Tecnológico (CNPq) pela concessão da bolsa de doutorado.

\section{REFERÊNCIAS}

AKAIKE, H. A new look at the statistical model identification. IEEE Transactions on Automatic Control, Boston, v. 19, n. 6, p. 716 - 723, 1974.

BOX, G. E. P.; COX, D. R. An Analysis of Transformations. Journal of the Royal Statistical Society, London, v. 26, n. 2, p. 211 - 252, 1964. 
BONAT, W. H.; RIBEIRO JÚNIOR, P. J.; ZEVIANI, W. M. Comparando predições por modelos geoestatístico e aditivo generalizado para reconstituição de superfícies contínuas gaussianas. Energia na Agricultura, v. 26, n. 2, p. 119 - 128, 2011.

DEMIDENKO, E. Mixed Models: theory and applications with R. 2 ${ }^{\mathrm{a}}$ ed. Canada: Willey, 2013. 637 p.

DIGGLE, P. J.; RIBEIRO JUNIOR, P. J. Model-Based geostatistics. New York: Springer, 2007. 228 p.

GREGOIRE, T. G.; SCHABENBERGER, O. Fitting bole-volume equations to spatially correlated within-tree data. In: ANNUAL CONFERENCE OF APPLIED STATISTICS IN AGRICULTURE, 6. 1994, Manhattan. Proceedings... Kansas, 1994. p. 120 - 133.

KITANIDIS, J. K. Introduction to geostatistics: applications in hydrology. New York: Cambridge University Press, 1997. 247 p.

MATÈRN, B. Spatial Variation. Berlin: Springer, 1986. 594 p.

MILLAR, R. B. Simulated maximum likelihood applied to non-Gaussian and nonlinear mixed effect and state-space models. Australian and New Zealand Journal of Statistics, v. 46, issue 4, p. 543 - 544, 2004.

MORENO, J. A. Clima do Rio Grande do Sul. Porto Alegre: Secretaria da Agricultura, 1961. 73 p.

PÉLISSIER, R.; GOREAUD, F. A practical approach to the study of spatial structure in simple cases of heterogeneous vegetation. Journal of Vegetation Science, v.12, issue 1, p. 99 - 108, 2001.

PLONER, A.; DUTTER, R. New directions in geostatistics. Journal of Statistical Planning and Inference, Amsterdam, v. 91, p. 499 - 509, 2000.

PRODAN, M. Holzmesslehre. Frankfurt, J. D. Sauerländer's Verlag, 1965. 644 p.

R CORE TEAM. R: A language and environment for statistical computing. $R$ Foundation for Statistical Computing, Vienna, Austria: R Foundation for Statistical Computing, 2013. Disponível em: <http://www.R-project.org/>. Acesso em: 12 abr. 2014.

REITZ, R.; KLEIN, R. M.; REIS, A. Projeto madeira do Rio Grande do Sul. Porto Alegre: CORAG, 1988. $525 \mathrm{p}$.

RIBEIRO JÚNIOR, P. J.; DIGGLE, P. J. The geoR package functions for geostatistical data analysis: R. News, v. 1, n. 2, p. 15 - 18, 2001.

RODE, R.; FIGUEIREDO FILHO, A.; MACHADO, S. A.; GALVÃO, F. Análise do padrão espacial de espécies e de grupos florísticos estabelecidos em um povoamento de Araucária angustifólia e em uma Floresta Ombrófila Mista no Centro-Sul do Paraná. Floresta, Curitiba, v. 40, n. 2, p. 255 - 268, 2010.

SCHNEIDER, P. R.; FINGER, C. A. G. Manejo Sustentado de Florestas Inequiâneas Heterogêneas. Santa Maria: UFSM, 2000. 195 p.

UNIVERSIDADE FEDERAL DE SANTA MARIA (UFSM). Inventário florestal contínuo do Rio Grande do Sul. Santa Maria: Convênio UFSM/SEMA, 2001. 706 p. (Relatório final original).

VACCARO, S. Caracterização fitossociológica de três fases sucessionais de uma Floresta Estacional Decidual, no município de Santa Tereza, RS. 92 f. Dissertação (Mestrado em Engenharia Florestal). Centro de Ciências Florestais, UFSM, Santa Maria, 1997.

VIEIRA, S. R.; LOMBARDI NETO, F. Variabilidade espacial do potencial de erosão das chuvas do Estado de São Paulo. Bragantina, Campinas, v. 54, n. 2, p. 405 - 412, 1995.

VUOLO, J. H. Fundamentos da Teoria de Erros. 2. ed. São Paulo: Edgar Blücher, 1996. 247 p.

WU, L. Mixed Effects Models for Complex Data. Canada: Tylor \& Francis Group, 2010. 419 p. 